\title{
ON SIGMA-IDEALS OF SETS 1
}

\author{
C. G. MENDEZ
}

\begin{abstract}
Let $\Phi(\Psi)$ denote the family of subsets of the unit square defined to be of first category (Lebesgue measure zero) in almost every vertical line in the sense of measure (category). THEOREM 1. (i) $\Phi$ and $\Psi$ are o-ideals. (ii) The union of $\Phi$ or $\Psi$ is $I \times I$. (iii) The complement of each member of $\Phi$ or $\Psi$ contains a set of power $c$ belonging to $\Phi$ and $\Psi$, respectively. (iv) The unit square may be represented as the union of two complementary Borel sets: one in $\Phi$ and $\Psi$ and the other one of Lebesgue measure zero and first category. (v) The unit square may be represented as the union of two complementary Borel sets: one in $\Phi$ and the other one in $\Psi$. THEOREM 2. $\Phi(\Psi)$ does not satisfy (vi) There is a subclass $\Upsilon$ of power $\leqslant c$ of the class $\Phi(\Psi)$ such that every member of the class is contained in some member of the subclass. THEOREM 3. There does not exist a one-to-one mapping $f$ from $I \times I$ onto itself, such that $K \in \Phi(\Psi)$ iff $f(K)$ is a Lebesgue measure zero (first category) subset of $I \times I$. Theorems 2 and 3 hold for more general $\Phi(\Psi)$.

A theorem on the theory of quotient (Boolean) algebras follows from these results.
\end{abstract}

1. On the Sierpinski-Erdös duality theorem. Let $\Phi$ denote the family of subsets of the unit square defined to be of first category in almost every vertical line in the sense of measure. To say in almost every vertical line in the sense of measure is equivalent to the statement: every vertical line except for a set whose corresponding projections to the $x$-axis is of measure zero in the unit interval. Let $\Psi$ denote the family of subsets of the unit square defined to be of measure zero in almost every vertical line in the sense of category. To say in almost every vertical line in the sense of category is equivalent to the statement: every vertical line except for a set whose corresponding projections to the $x$-axis is of first category in the unit interval. That is,

1.1 Definition. $K \in \Phi(\Psi)$ iff there is a measure zero (first category) set $S$ in $I$ (where $I$ denotes the unit interval) such that $K_{x}$ is of first category (measure zero for every $x$ in $I-S$, (where $K_{x}=\{y \in I:(x, y) \in K$, for some fixed $x$ in $I\}$ ).

Without gain of generality the roles of vertical line and $x$-axis could have been interchanged with those of horizontal line and $y$-axis, respectively,

Presented to the Society, January 21, 1976 in part under the title On the Sierpiniski-Erdös duality theorem; received by the editors March 25, 1976.

AMS (MOS) subject classifications (1970). Primary 28A05, 28A10, 54A05, 04A05, 04A15, 54C50, 54H05, 54H99; Secondary $02 \mathrm{~J} 04$.

Key words and phrases. Measure zero, first category, $\sigma$-ideals of sets, set theoretically equivalent classes of sets, continuum, Sierpiński-Erdös duality theorem, quotient (Boolean) algebras.

1 These results are contained in the author's Ph.D. dissertation submitted to the University of Colorado, Boulder, in December of 1974 and written under the direction of Professor S. M. Ulam. 
Throughout this article measure zero is to mean Lebesgue measure zero unless otherwise stated.

1.2 ThEOREM. (i) $\Phi$ and $\Psi$ are $\sigma$-ideals.

(ii) The union of $\Phi$ or $\Psi$ is $I^{2}$ (where $I^{2}=I \times I$ ).

(iii) The complement of each member of $\Phi$ or $\Psi$ contains a set of power $c$ belonging to $\Phi$ or $\Psi$, respectively.

(iv) The unit square may be represented as the union of two complementary Borel sets one in $\Phi$ and $\Psi$ and the other one of measure zero and of first category.

(v) The unit square may be represented as the union of two complementary Borel sets one in $\Phi$ and the other one in $\Psi$.

Proof. (i) Straightforward.

(ii) $I^{2}$ is the union of its singleton subsets.

(iii) $K \in \Phi \Rightarrow K_{x}$ is of first category for almost every $x$ and hence for some $x$.

Now, let $I^{2}(x)=\left\{(x, y) \in I^{2}\right.$, for some fixed $\left.x \in I\right\}$ and let $K(x)=I^{2}(x)$ $\cap K$. Then $I^{2}(x)-K(x)$ is of second category in $I^{2}(x)$ for such $x$ (by Baire's category theorem) and hence of power $c$. Needless to say $I^{2}(x)-K(x) \in \Phi$ and is contained in the complement of $K$.

Similarly, for $\Psi$.

(iv) and (v) I may be represented as the union of two complementary sets $A$ and $B$ such that $A$ is an $F_{\sigma}$ set of first category and $B$ is a $G_{\sigma}$ set of measure zero [4, p. 5]. Hence $I^{2}$ may be represented as the union of two complementary Borel sets $I \times A$ and $I \times B$ where $I \times A \in \Phi$ and of first category ([4, p. 57]) and $I \times B \in \Psi$ and of measure zero $([4$, p. 54]).

Moreover, $I^{2}=((A \times A) \cup(B \times B)) \cup((B \times A) \cup(A \times B))$ where $(A$ $\times A) \cup(B \times B)$ is of first category in almost every vertical line in the sense of measure, and of measure zero in almost every vertical line in the sense of category. That is, $(A \times A) \cup(B \times B) \in \Phi$ and $\Psi$.

On the other hand, $(B \times A) \cup(A \times B)$ is of first category in almost every vertical line in the sense of category and hence of first category $([4, \mathrm{p} .57])$; as well as of measure zero in almost every vertical line in the sense of measure and hence of measure zero $([4$, p. 54]).

That is, $(B \times A) \cup(A \times B)$ is of first category and of measure zero. Q.E.D.

It is well known that the classes of measure zero and first category sets satisfy (i)-(iii) of 1.2 Theorem together with a corresponding property (v). Moreover, each of them satisfies the following additional property:

(vi) There is a subclass $\Upsilon$ of power $\leqslant c$ of the class of measure zero (or first category) sets such that every member of the class is contained in some member of the subclass $\Upsilon([2$, pp. 5,15$],[4$, p. 77]).

Assuming the continuum hypothesis, a purely set theoretical argument is then sufficient to establish the Sierpiński-Erdös duality theorem for the classical $\sigma$-ideals $([4$, p. 76$])$. 
Our study of 1.2 Theorem was primarily motivated as an attempt to establish a corresponding property (vi) for $\Phi$ and $\Psi$. However, using a diagonal argument we shall show that there is no subclass $\Upsilon$ of power $\leqslant c$ of the class $\Phi(\Psi)$ such that every member of $\Phi(\Psi)$ is contained in some member of the subclass $\Upsilon$. And hence that the desired theorem cannot be established for $\Phi(\Psi)$. Instead, we have 1.4 Theorem.

\subsection{THEOREM. $\Phi(\Psi)$ do not satisfy property (vi).}

Proof. Suppose otherwise. That is, let us assume that there is a subclass $\Gamma=\left\{S_{1}, S_{2}, S_{3}, S_{4}, \ldots, S_{n}, \ldots, S_{\xi}, \ldots, \xi<c\right\}$ of $\Phi$ of power $c$ such that every member of $\Phi$ is contained in some member of the subclass $\Gamma$.

And let us consider the following inductively defined one-to-one correspondence $f$ from $\Gamma$ into $I$ :

Given any $S_{\xi} \in \Gamma$ there is a corresponding $x_{\xi}=f\left(S_{\xi}\right)$ in $I$ such that $x_{\xi} \neq x_{\alpha}$ for all $\alpha<\xi$ and $\left(S_{\xi}\right)_{x_{\xi}}=\left\{(x, y) \in S_{\xi}: x=x_{\xi}\right\}$ is a set of first category. This is possible for $\left\{x_{\alpha}: \alpha<\xi<c\right\}$ is of power $<c$ and $\left(S_{\xi}\right)_{x}$ is a set of first category for almost every $x$ in the sense of measure, i.e., $\left(S_{\xi}\right)_{x}$ is a set of first category for every $x$ in $I-S$ where $S$ is a set of measure zero; and hence, for a continuum many $x$ (for $I-S$ is of power $c([4$, p. 74])).

At this point we shall proceed to define a set $K$ in $\Phi$ such that $K$ is not contained in any member of $\Gamma . K$ may be chosen to be any set satisfying the following conditions:

(a) Given any $S_{\xi} \in \Gamma$, and $x_{\xi}=f\left(S_{\xi}\right)$, let $K\left(x_{\xi}\right)=\left\{\left(x_{\xi}, y_{\xi}\right)\right\}$ where $\left\{\left(x_{\xi}, y_{\xi}\right)\right\}$ is any singleton in $I^{2}\left(x_{\xi}\right)$ not contained in $S_{\xi}\left(x_{\xi}\right)$. This is possible for $\left(S_{\xi}\right)_{x_{\xi}}$ is of the first category and by Baire's category theorem is not equal to $I$.

(b) $K(x)$ is of the first category for all other $x$.

Hence, $K$ is not contained in any $S_{\xi} \in \Gamma$ (by (a)). $K_{x}$ is of first category for every $x$ (by (a) and (b)) $\Rightarrow K \in \Phi$.

A similar argument for $\Psi$ shall be omitted. Q.E.D.

1.4 THEOREM. There does not exist a one-to-one mapping from $I^{2}$ onto $I^{2}$ such that $K \in \Phi(\Psi)$ iff $f(K)$ is a measure zero (or first category) subset of $I^{2}$.

Proof. Suppose $f$ is such a mapping. Let $\Gamma$ be the class of $G_{\delta}$ sets of measure zero in $I^{2}$. Then $f^{-1}(\Gamma)$ is a class of power $c$ that generates $\Phi$, contrary to 1.3 Theorem. Similarly, for $\Psi$, when $\Gamma$ is taken to be the class of $F_{\sigma}$ sets of first category. Q.E.D.

1.5 REMARK. In view of 1.4 Theorem, we would naturally like to determine whether $\sigma$-homomorphisms $([6$, p. 82]) of $\sigma$-ideals are always generated by point mappings ([5, p. 7]); for clearly, an affirmative response to this question would insure that no $\sigma$-homomorphism may be established between the $\sigma$ ideals under consideration, and hence that the Sierpiński-Erdös duality principle $([4$, p. 75$])$ would fail for $\Phi(\Psi)$ and the class of sets of measure zero, or the class of sets of first category.

However, it is my understanding that the proposed problem is still open. 
On the other hand, we have:

1.6 THEOREM. Assuming the continuum hypothesis, the Sierpiński-Erdös theorem is satisfied for $\Phi$ and $\Psi$.

Proof. Let $f$ be the Sierpiński-Erdös one-to-one mapping from $I$ onto $I$ such that $f=f^{-1}$ and $K$ is a measure zero subset of $I$ iff $f(K)$ is a first category subset of $I\left([4\right.$, p. 77] $)$. Then $f \times f$ is a one-to-one mapping from $I^{2}$ onto $I^{2}$ such that $f \times f=(f \times f)^{-1}$ and $K \in \Phi$ iff $f(K) \in \Psi$. Q.E.D.

1.7 REMARK. The results of this section may be extended (under the necessary assumptions) as follows:

Let $\Delta$ and $\nabla$ denote any two $\sigma$-ideals of two arbitrary sets $X$ and $Y$, respectively; and let us define $\Phi$ to be the family of subsets $K$ of $X \times Y$ such that $K_{x}$ is in $\nabla$ for almost all $x$ in the sense of $\Delta$.

How about defining $\Phi(\Psi)$ to be the class of all subsets of $\prod_{i=1}^{n} X_{i}$ (for arbitrary sets $X_{i}$ ) of first category (measure zero) in almost every (vertical) line $l_{x_{i}}: l_{x_{i}}=\left\{\left(x_{1}, x_{2}, \ldots, x_{i}, \ldots, x_{n}\right) \in \prod_{i=1}^{n} X_{i}\right.$, for some fixed $i$ and $\left.x_{i} \in X_{i}\right\}$ in the sense of measure (category); or more generally, to be the class of all subsets of $\prod_{i=1}^{n} X_{i}$ of first category in almost every $n$-dimensional (orthogonal) hyperplane (or, every set in an arbitrary class of subsets of $\prod_{i=1}^{n} X_{i}$ ) in the sense of some measure defined for such a class?

It is feasible and/or productive to explore any or all of these ideas for infinitely many dimensions; i.e., on Hilbert, and/or Banach spaces?

One may also consider defining $\Phi(\Psi)$ to be the class of all subsets on the unit square, or $X \times X$ (for an arbitrary set $X$ ) ([3, p. 1-39]) of first category (measure zero) in almost every line in the sense of measure (category) (provided that we may assume the existence of a measure in the set of all lines under consideration).

2. On quotient sigma-algebras. We shall establish in this section a couple of results on the theory of Boolean algebras which follow from our development above.

2.1 THEOREM. (i) $\Xi / \Theta$ and $\Xi / \Phi(\Psi)$ are not isomorphic.

(ii) $\Xi / \Pi$ and $\Xi / \Phi(\Psi)$ are not isomorphic,

(iii) $\Gamma / \Phi$ and $\Gamma / \Psi$ are isomorphic (assuming the continuum hypothesis); where $\Phi$ and $\Psi$ are as defined above, $\Theta$ denotes the class of measure zero sets, and $\Pi$ the class of sets of first category; while $\Xi$ and $\Gamma$ denote the families of all Borel subsets, and all subsets of $I^{2}$, respectively.

Proof. (i) There is a nonzero $\sigma$-finite measure $\mu$ on $\Xi / \Theta$, i.e., the measure $\mu$ induced in the natural way by the Lebesgue measure $m$ such that $\mu([A])$ $=m(A)$ for all Borel $A$ in $I^{2}$. On the other hand, every $\sigma$-finite measure on $\Xi / \Phi(\Psi)$ vanishes identically. In fact, every $\sigma$-finite measure on $\Xi / \Phi(\Psi)$ determines a $\sigma$-finite measure on $\Xi$ as illustrated above and this measure $\mu$ vanishes on all sets $A$ in $\Phi(\Psi)$. Since $I^{2}$ may be represented as the union of two complementary Borel sets $A$ and $B$ where $A$ is in $\Phi(\Psi)$ and $B$ is of measure 
zero, we have:

$$
\mu\left(\left[I^{2}\right]_{\Phi(\Psi)}\right)=\mu\left([B]_{\Phi(\Psi)}\right)+\mu\left([A]_{\Phi(\Psi)}\right)=m(B)+m(0)=0 .
$$

Hence $\mu$ vanishes identically [1].

Moreover, since the existence of a $\sigma$-finite measure on a Boolean $\sigma$-algebra is invariant under isomorphism, we infer that $\Xi / \Theta$ and $\Xi / \Phi(\Psi)$ are not isomorphic $([6$, p. 73]).

(ii) Since $I^{2}$ is a complete separable metric space, then every isomorphism of $\Xi / \Pi$ onto $\Xi / \Phi(\Psi)$ is induced by a point mapping $([6$, p. 139]).

And since such a point mapping is a Sierpiński mapping [4] from $\Pi$ onto $\Phi(\Psi)$, we infer that such an isomorphism is not possible, for there is no such Sierpiński mapping (by 1.4).

(iii) There is a Sierpiński mapping $f$ from $\Phi$ onto $\Psi$ (cf. 1.6). Hence $h\left([A]_{\Phi}\right)=[f(A)]_{\Psi}$, for $A$ in $\Gamma$, defines an isomorphism $h$ of $\Gamma / \Phi$ onto $\Gamma / \Psi$. Q.E.D.

\section{REFERENCES}

1. A. Horn and A. Tarski, Measures in Boolean algebras, Trans. Amer. Math. Soc. 64 (1948), 467-497. MR 10, 518.

2. C. G. Mendez, The analogy between the concepts of first category and measure zero, M.A. Thesis, Univ. of Colorado, 1968.

3. - On Borel and analytic structures and the interrelation of measure and category in an abstract setting. On sigma-ideals of sets and on measures in $2^{x}, \mathrm{Ph} . \mathrm{D}$. Dissertation, Univ. of Colorado, 1974.

4. J. C. Oxtoby, Measure and category, Springer-Verlag, New York, 1971.

5. R. Sikorski, On the inducing of homomorphisms by mappings, Fund. Math. 36 (1949), 7-22. MR 11, 166.

6. - Boolean algebras, 2nd ed., Academic Press, New York, 1964. MR 31 \#2178.

Department of Mathematics, Metropolitan State College, Denver, Colorado 80204 